\title{
Adaptive Neural Tracking Control for a Two-Joint Robotic Manipulator with Unknown Time-Varying Delays
}

\author{
Jiayao Wang $(\mathbb{D}$ and Yang Cui $(i D)$ \\ School of Electronic and Information Engineering, University of Science and Technology Liaoning, Anshan 114051, China \\ Correspondence should be addressed to Yang Cui; cuiyangfly@sina.com
}

Received 28 August 2021; Revised 5 December 2021; Accepted 16 December 2021; Published 25 January 2022

Academic Editor: Honglei Xu

Copyright (@ 2022 Jiayao Wang and Yang Cui. This is an open access article distributed under the Creative Commons Attribution License, which permits unrestricted use, distribution, and reproduction in any medium, provided the original work is properly cited.

\begin{abstract}
This paper presents an adaptive neural tracking control approach for a two-joint robotic manipulator with unknown time-varying delays. In order to work out the effect of unknown time-varying delays on the two-joint robotic manipulator, the appropriate Lyapunov-Krasovskii functionals (LKFs) and separation technology are chosen to settle this matter. The neural networks work as an approximator that has the advantage of estimating the unknown function in the system. In this paper, Lyapunov stability analysis can prove that all signals of the closed-loop system are semiglobal uniformly ultimately bounded and the tracking error can converge to a compact neighborhood with respect to zero. The simulation consequences demonstrate the availability of the feedforward control approach.
\end{abstract}

\section{Introduction}

Recently, the adaptive backstepping control method has been used in a wide range of applications in control field, such as marine vessels control [1], autonomous underwater vehicle system [2], and aircraft flight control system [3]. The adaptive control method is principally worked out for the nonlinear system with uncertain linear parameters. However, there are many nonlinear systems with uncertainty that cannot be linearly parameterized. For the sake of settling this difficulty, these uncertainties are always approximated by some universal approximators in the literature [4-9], such as fuzzy logic systems and neural networks. In $[10,11]$, the tracking controllers are designed based on adaptive neural/fuzzy backstepping control methods and the tracking error can converge to the accuracy defined a priori in probability. In the literature $[12,13]$, the adaptive neural/fuzzy control strategies are proposed that aim to approximate unknown function for the nonlinear multiple-input and multiple-output (MIMO) system. The traditional adaptive neural/fuzzy backstepping control methods have great development, but with the increase of system order, the structure of the controller is complex.
For the traditional backstepping controller design process of the system, the controller design in each step requires to take the derivative of the previous virtual controller in [14-16]; the controller design process will be more complicated when the order of the system is higher, and there will be appeared "explosion of complexity" phenomenon. To work out the "explosion of complexity" problem, the command filtering technique is used in the traditional backstepping process to eliminate the repeated differentiation of the virtual controller in [17-21]. In the literature [22, 23], the authors have proposed improved control methods by designing compensation signals, which can eliminate filter errors caused by command filters. In the meantime, the singularity problem is a possible problem in controller design process; however, this point was not considered in the abovementioned literature. Therefore, this paper will consider command filtering techniques and study the singularity problems that may occur in the controller design process.

Time delays are common in the practical application of control. The problem of time delays in the system will greatly reduce the control performance. However, the abovementioned articles do not consider the issue of time delays. In order to solve this problem, many scholars and experts have shown interest in solving the problem of time delays. LKFs are effective tools to 
restrain the influence of time delays in nonlinear systems [24-28]. In the literature [24-27], the authors use LKFs and backstepping method to solve the problem of the nonlinear system with time-varying delay. In [28], an adaptive neural tracking controller is constructed by using LKFs and separation techniques for MIMO nonlinear systems with constant time delays. Inspired by the above literature, the method of combining LKFs and separation technology will be utilized to solve the time-varying delay.

Due to the industry being developed increasingly more rapidly, robot manipulators have been widely applied in the production. More recently, the robotic manipulator has been a research hotspot and some achievements have been made in [29-31]. In the literature [32, 33], based on the barrier Lyapunov functions, the adaptive neural/fuzzy control methods are proposed for the manipulator with full state constraints. By using auxiliary design system, an adaptive impedance controller is designed for the manipulator system with input saturation in [34]. The adaptive control methods proposed in [29-34] can effectively solve the control problem of the robotic manipulator without time-varying delays. When using a robotic manipulator to solve actual industrial problems, due to signal transmission and other reasons, time delay is inevitable. Therefore, it is of theoretical and practical significance to study the adaptive tracking control problem of a robotic manipulator with time-varying delays.

Enlightened by the above results, based on the backstepping control method, we will employ neural networks, command filtering technology, LKFs, and the separation techniques to achieve the tracking control objective of the robotic manipulator with unknown time-varying delays. In the meantime, this paper will take into account the singularity problem that may occur in the controller design process. The contributions of this paper are stated as follows:

(1) For the traditional backstepping control method, there exists the repeated derivative phenomenon of the virtual controller in [14-16]. In order to avoid the derivation of the virtual controller, we choose the command filtering control method to solve this problem.

(2) In the literature [35], the authors have adopted L'Hopital's rule to effectively avoid the occurrence of the singularity problem. However, L'Hopital's rule must meet the following conditions before it can be used: (1) The limits of the numerator and denominator are both equal to zero or infinity. (2) The numerator and denominator are, respectively, differentiable within the restricted region. However, since the situation of this paper is different from that in the literature [35], this paper further considers the case that the numerator and denominator are not zero at the same time when the singularity problem occurs. Then, we propose a piecewise function method to solve this singularity problem.

(3) The adaptive neural controllers of the robot manipulator without time-varying delay were investigated in the literature $[32,34]$. But, in fact, timevarying delays are often encountered in practical engineering systems. In this paper, we consider studying a novel control method of the robot manipulator with time-varying delays and the unknown timevarying delays were solved by the separation technology and LKFs. The separation technique shows advantage of breaking up the time-varying delay functions to several continuous known functions, which are eliminated by making use of the LKFs.

The structure of this article is shown as follows: In Section 2, the two-joint robot manipulator with time-varying delays is introduced. The adaptive neural controller design and stability analysis are performed in Section 3. In Section 4, the availability of the proposed control method is proved by a simulation. Section 5 is the conclusion of this paper.

\section{System Descriptions and Preliminaries}

The dynamic model for the two-joint robot manipulator with unknown time-varying delays is expressed as

$$
\begin{aligned}
& M(q) \ddot{q}+C(q, \dot{q}) \dot{q}+T\left(q\left(t-\vartheta_{1}(t)\right), \dot{q}\left(t-\vartheta_{2}(t)\right)\right)+G(q) \\
& \quad=u-J^{T}(q) p(t),
\end{aligned}
$$

where $q=\left[q_{1}, q_{2}\right]^{T}, \dot{q}$, and $\ddot{q} \in R^{2}$ are the angular location, speed, and acceleration vectors; $\vartheta_{1}(t)$ and $\vartheta_{2}(t)$ stand for the unknown time-varying delays; $\vartheta_{1}(t)$ and $\vartheta_{2}(t)$ satisfied $\vartheta_{i}(t) \leq \vartheta_{\max }$ and $\vartheta_{i}(t) \leq \vartheta \leq 1$, where $\vartheta_{\max }$ and $\vartheta$ are the known constants; $u$ presents the applied torque; $p(t)$ stands for the restraining force and satisfies $|p(t)| \leq \bar{p}$ for $t>0$, where $\bar{p}$ is a positive constant; $M(q)$ represents the symmetric positive definite inertia matrix; $C(q, \dot{q}) \dot{q}$ stands for the unknown centripetal and Coriolis torque; $T(\cdot)$ stands for the unknown time delay function; $G(q)$ is the unknown gravitational force; and $J^{T}(q)$ represents the unknown reversible Jacobian matrix.

For the states defined as $x_{1}=\left[q_{1}, q_{2}\right]^{T}$ and $x_{2}=\left[\dot{q}_{1}, \dot{q}_{2}\right]^{T}$, respectively, the state-space model of the dynamic model for the two-joint robot manipulator system can be rewritten as

$$
\left\{\begin{array}{l}
\dot{x}_{1}=x_{2}, \\
\dot{x}_{2}=M^{-1}\left(x_{1}\right)\left(-C\left(x_{1}, x_{2}\right) x_{2}-G\left(x_{1}\right)-J^{T}\left(x_{1}\right) p(t)\right. \\
\left.-T\left(x_{1}\left(t-\vartheta_{1}(t)\right), x_{2}\left(t-\vartheta_{2}(t)\right)\right)+u\right), \\
y_{1}=x_{1} .
\end{array}\right.
$$

In this paper, the control objective is to design an adaptive neural tracking controller based on backstepping technique for systems (1), so that the angular position $y_{1}=x_{1}=\left[q_{1}, q_{2}\right]^{T}$ can track the reference signal $y_{r}$ and all signals of the closed-loop system are semiglobal uniformly ultimately bounded.

Notation 1 (see [34]). The matrix $2 C(q, \dot{q})-\dot{M}(q)$ represents skew-symmetric when matrix $C$ has an appropriate definition.

Notation 2 (see [36]). The matrix $M(q)$ stands for symmetric and positive definite.

Assumption 1 (see [37]). The first and second derivatives of the reference signal $y_{r}$ are continuous and bounded. 
Assumption 2 (see $[38,39]$ ). For the unknown and smooth nonlinear function $T(\cdot)$ satisfying $|T(\cdot)| \leq \bar{T}(\cdot)$, where $\bar{T}(\cdot)$ is a positive and smooth function, we can obtain the following inequality:

$$
\begin{aligned}
& \left\|T\left(x_{1}\left(t-\vartheta_{1}(t)\right), x_{2}\left(t-\vartheta_{2}(t)\right)\right)\right\| \\
& \quad \leq \bar{T}\left(x_{1}\left(t-\vartheta_{1}(t)\right), x_{2}\left(t-\vartheta_{2}(t)\right)\right) .
\end{aligned}
$$

Assumption 3 (see $[38,39])$. There exist positive and smooth functions $q_{1}(\cdot)$ and $q_{2}(\cdot)$ that are the upper bound of the time-varying delay function $\bar{T}(\cdot)$; the following inequality holds:

$$
\begin{aligned}
\bar{T}\left(x_{1}\left(t-\vartheta_{1}(t)\right), x_{2}\left(t-\vartheta_{2}(t)\right)\right) \leq & q_{1}\left(x_{1}\left(t-\vartheta_{1}(t)\right)\right)\left\|x_{1}\left(t-\vartheta_{1}(t)\right)\right\| \\
& +q_{2}\left(x_{1}\left(t-\vartheta_{1}(t)\right), x_{2}\left(t-\vartheta_{2}(t)\right)\right)\left\|x_{2}\left(t-\vartheta_{2}(t)\right)\right\| .
\end{aligned}
$$

Remark 1. It is noticed that Assumptions 1-3 are the standard assumptions for tracking control of the system with unknown time-varying delays and similar assumptions can be found in [37-39]. Without these assumptions, the proposed scheme cannot be realized.

Remark 2. How to eliminate the unknown function $T\left(x_{1}\left(t-\vartheta_{1}(t)\right), x_{2}\left(t-\vartheta_{2}(t)\right)\right)$ with the time-varying delays $\vartheta_{1}(t)$ and $\vartheta_{2}(t)$ is the key to solving the problem. To settle this matter, this paper adopts the separation technique that disintegrates the unknown time-varying delays function $T(\cdot)$ to a number of positive and continuous functions and applies the appropriate LKFs to compensate that. In the following part, in order to convince the statement, the functions $q_{1}\left(x_{1}\left(t-\vartheta_{1}(t)\right)\right)$ and $q_{2}\left(x_{1}\left(t-\vartheta_{1}(t)\right)\right.$, $\left.x_{2}\left(t-\vartheta_{2}(t)\right)\right)$ are simplified to $q_{1}$ and $q_{2}$.

\section{Controller Design and Stability Analysis}

Denote the tracking error signals as

$$
\begin{aligned}
& z_{1}=x_{1}-y_{r}, \\
& z_{2}=x_{2}-s_{2},
\end{aligned}
$$

where $s_{2}$ stands for the filter output.

Denote the compensating tracking error as

$$
\begin{aligned}
& \breve{z}_{1}=z_{1}-r_{1}, \\
& \breve{z}_{2}=z_{2}-r_{2},
\end{aligned}
$$

where $r_{1}$ and $r_{2}$ are the compensating signals.

Then, the derivative of $\breve{z}_{1}$ can be expressed as

$$
\dot{\check{z}}_{1} \cdot=x_{2}-\dot{y}_{r}-\dot{r}_{1} \text {. }
$$

We choose

$$
\dot{r}_{1}=-k_{1} r_{1}+r_{2}+s_{2}-s_{2}^{0}
$$

where $k_{1}=\operatorname{diag}\left\{k_{11}, k_{12}\right\}$ and $k_{1 i}, i=1,2$, represent the positive constant. $s_{2}^{0}$ stands for the virtual controller that will be defined later.

Substituting (8) into (7) gives

$$
\dot{z}_{1}=\breve{z}_{2}-\dot{y}_{r}+k_{1} r_{1}+s_{2}^{0} .
$$

Design the virtual control law as

$$
s_{2}^{0}=-k_{1} z_{1}+\dot{y}_{r} \text {. }
$$

From (10), (9) can be obtained as

$$
\dot{\check{z}}_{1}=-k_{1} \breve{z}_{1}+\breve{z}_{2} \text {. }
$$

Choose a Lyapunov function candidate in the following:

$$
V_{1}=\frac{1}{2} \bar{z}_{1}^{T} \breve{z}_{1}
$$

Taking the derivative of $V_{1}$ yields

$$
\begin{aligned}
\dot{V}_{1} & =\breve{z}_{1}^{T} \dot{z}_{1} \\
& =-\sum_{i=1}^{2} k_{1 i} \breve{z}_{1 i}^{2}+\breve{z}_{1}^{T} \breve{z}_{2} .
\end{aligned}
$$

The derivative of $\breve{z}_{2}$ can be described as

$$
\dot{\bar{z}}_{2}=\dot{x}_{2}-\dot{s}_{2}-\dot{r}_{2} \text {. }
$$

We choose

$$
\dot{r}_{2}=-k_{2} r_{2}-r_{1} \text {. }
$$

Substituting (15) into (14), we can get

$$
\dot{\bar{z}}_{2}=\dot{x}_{2}-\dot{s}_{2}+k_{2} r_{2}+r_{1} \text {. }
$$

Consider the state-space model of system (2), (16) can be changed as

$$
\begin{aligned}
\dot{\vec{z}}_{2}= & M^{-1}\left(x_{1}\right)\left(-C\left(x_{1}, x_{2}\right) x_{2}-G\left(x_{1}\right)-J^{T}\left(x_{1}\right) p(t)\right. \\
& \left.+u-T\left(x_{1}\left(t-\vartheta_{1}(t)\right), x_{2}\left(t-\vartheta_{2}(t)\right)\right)\right)-\dot{s}_{2}+k_{2} r_{2}+r_{1} .
\end{aligned}
$$


Choose a Lyapunov function candidate from the following:

$$
V_{2}=V_{1}+\frac{1}{2} \bar{z}_{2}^{T} M\left(x_{1}\right) \breve{z}_{2}+\sum_{i=1}^{2} \frac{1}{2 \beta} \widetilde{W}_{i} \Gamma_{i}^{-1} \widetilde{W}_{i},
$$

where $\widetilde{W}_{i}=W_{i}^{*}-\widehat{W}_{i}$ represents the weight estimation error and $\beta$ stands for the positive constant.
Then, the derivative of $V_{2}$ can be expressed as

$$
\dot{V}_{2}=-\sum_{i=1}^{2} k_{1 i} \breve{z}_{1 i}^{2}+\breve{z}_{1}^{T} \breve{z}_{2}+\breve{z}_{2}^{T} M\left(x_{1}\right) \breve{z}_{2}-\sum_{i=1}^{2} \frac{1}{\beta} \widetilde{W}_{i} \Gamma_{i}^{-1} \dot{\hat{W}}_{i} \text {. }
$$

Substituting (17) into (19) gives

$$
\begin{aligned}
\dot{V}_{2}= & -\sum_{i=1}^{2} k_{1 i} \breve{z}_{1 i}^{2}+\breve{z}_{1}^{T} \breve{z}_{2}+\breve{z}_{2}^{T}\left(-C\left(x_{1}, x_{2}\right) x_{2}-G\left(x_{1}\right)-J^{T}\left(x_{1}\right) p(t)+M\left(x_{1}\right)\left(-\dot{s}_{2}+k_{2} r_{2}+r_{1}\right)\right) \\
& +\breve{z}_{2}^{T} u-\sum_{i=1}^{2} \frac{1}{\beta} \widetilde{W}_{i} \Gamma_{i}^{-1} \dot{\hat{W}}_{i}-\breve{z}_{2}^{T} T\left(x_{1}\left(t-\vartheta_{1}(t)\right), x_{2}\left(t-\vartheta_{2}(t)\right)\right) .
\end{aligned}
$$

Combining Young's inequalities with Assumption 2, we can get where $\varrho=1-\vartheta$ is a positive constant.

Based on (21), (20) is rewritten as

Denote the unknown function $H(Z)$ as follows: According to (23), (22) can be changed as

$$
\begin{aligned}
H(Z)= & -C\left(x_{1}, x_{2}\right) x_{2}-G\left(x_{1}\right)+M\left(x_{1}\right)\left(-\dot{s}_{2}+k_{2} r_{2}+r_{1}\right) \\
& -J^{T}\left(x_{1}\right) p(t)+\frac{1}{\breve{z}_{2}^{T}} \sum_{k=1}^{2} \exp \left(\vartheta_{k}(t)\right)\left(q_{k}^{2}\left\|x_{k}(t)\right\|^{2}\right) .
\end{aligned}
$$

$$
\begin{aligned}
\dot{V}_{2} \leq & -\sum_{i=1}^{2} k_{1 i} \breve{z}_{1 i}^{2}+\breve{z}_{1}^{T} \breve{z}_{2}+\breve{z}_{2}^{T} H(Z)+\frac{1}{4 \varrho} \breve{z}_{2}^{T} \breve{z}_{2}-\sum_{k=1}^{2} \exp \left(\vartheta_{k}(t)\right)\left(q_{k}^{2}\left\|x_{k}(t)\right\|^{2}\right) \\
& +\breve{z}_{2}^{T} u+\varrho \bar{T}^{2}\left(x_{1}\left(t-\vartheta_{1}(t)\right), x_{2}\left(t-\vartheta_{2}(t)\right)\right)-\sum_{i=1}^{2} \frac{1}{\beta} \widetilde{W}_{i} \Gamma_{i}^{-1} \dot{\widehat{W}}_{i} .
\end{aligned}
$$

On the basis of the neural network approximation, the function $H(Z)$ can be approximated as

$$
H_{i}(Z)=W_{i}^{*} S_{i}(Z)+\varepsilon_{i}(Z),
$$

where $Z$ stands for the input vector; $W_{i}^{*}$ represents the optimal weight vector of the neural networks;
$S_{i}(Z)=\left[S_{i l_{1}}(Z), \ldots, S_{i l_{i}}(Z)\right]^{T}$ denotes Gaussian basis function vector; and $l_{i}$ represents the node number of the neural networks. Let $W^{*}=\operatorname{diag}\left\{W_{1}^{*}, W_{2}^{*}\right\}, \quad \widehat{W}=\operatorname{diag}$ $\left\{\widehat{W}_{1}, \widehat{W}_{2}\right\}$, and $S(Z)=\operatorname{diag}\left\{S_{1}(Z), S_{2}(Z)\right\} ; \bar{\varepsilon}_{i}$ denotes a positive constant, and it represents the upper bound of approximation error $\varepsilon_{i}(Z)$, which satisfies $\left|\varepsilon_{i}(Z)\right| \leq \bar{\varepsilon}_{i}$. 
Then, define the piecewise function as

$$
Y\left(\breve{z}_{2}\right)= \begin{cases}0, & \breve{z}_{2}=[0,0]^{T} \\ 1, & \text { otherwise. }\end{cases}
$$

In terms of application practice, the control performance of the system is optimal when $\breve{z}_{2}=[0,0]^{T}$. According to (19), it can be expressed as $\dot{V}_{2}=-\sum_{i=1}^{2} k_{1} \breve{z}_{1}^{T} \breve{z}_{1}-\sum_{i=1}^{2} 1 / \beta \widetilde{W}_{i} \Gamma_{i}^{-1} \dot{\widehat{W}}_{i}$. The above equation is rewritten as $\dot{V}_{2}=-\sum_{i=1}^{2} k_{1} \breve{z}_{1}^{T} \breve{z}_{1}$ when the neural networks are left out. Employing Barbalat's lemma can testify the stability when $\breve{z}_{2}=[0,0]^{T}$.
Remark 3. Noticing equation (23) when $\breve{z}_{2}=[0,0]^{T}$, the appearance of singularity problem leads to a major difficulty. L'Hopital's rule is not satisfied because the numerator and denominator are not guaranteed to be zero at the same time and neural networks cannot be used to approximate the function. In order to solve the singularity problem, the piecewise function method will be utilized to work out the difficulties in the process of controller design. In the meantime, it is worth noting that the tracking performance of the control system is better when $\breve{z}_{2}=[0,0]^{T}$.

The neural networks can be employed to approximate the unknown function $H(Z)$ when $\breve{z}_{2} \neq[0,0]^{T}$. Then, adding (25) into (24), we gain

$$
\begin{aligned}
\dot{V}_{2} \leq & -\sum_{i=1}^{2} k_{1 i} \breve{z}_{1 i}^{2}+\breve{z}_{1}^{T} \breve{z}_{2}+\sum_{i=1}^{2} \breve{z}_{2 i} W_{i}^{*} S_{i}(Z)+\sum_{i=1}^{2} \breve{z}_{2 i} \varepsilon_{i}(Z)+\frac{1}{4 \varrho} \breve{z}_{2}^{T} \breve{z}_{2}-\sum_{k=1}^{2} \exp \left(\vartheta_{k}(t)\right)\left(q_{k}^{2}\left\|x_{k}(t)\right\|^{2}\right) \\
& +\breve{z}_{2}^{T} u+\varrho \bar{T}^{2}\left(x_{1}\left(t-\vartheta_{1}(t)\right), x_{2}\left(t-\vartheta_{2}(t)\right)\right)-\sum_{i=1}^{2} \frac{1}{\beta} \widetilde{W}_{i} \Gamma_{i}^{-1} \dot{\widehat{W}}_{i} .
\end{aligned}
$$

According to Young's inequality, we can get

$$
\sum_{i=1}^{2} \breve{z}_{2 i} \varepsilon_{i}(Z) \leq \frac{1}{2} \sum_{i=1}^{2} \frac{1}{\eta} z_{2 i}^{2}+\frac{1}{2} \sum_{i=1}^{2} \eta \bar{\varepsilon}_{i}^{2},
$$

where $\eta$ represent positive constant.

Adding (28) into (27), we can obtain

$$
\begin{aligned}
\dot{V}_{2} \leq & -\sum_{i=1}^{2} k_{1 i} \breve{z}_{1 i}^{2}+\breve{z}_{1}^{T} \breve{z}_{2}+\sum_{i=1}^{2} \breve{z}_{2 i} W_{i}^{*} S_{i}(Z)+\frac{1}{2} \sum_{i=1}^{2} \frac{1}{\eta} \breve{z}_{2 i}^{2} \\
& +\frac{1}{2} \sum_{i=1}^{2} \eta_{i} \bar{\varepsilon}_{i}^{2}-\sum_{k=1}^{2} \exp \left(\vartheta_{k}(t)\right)\left(q_{k}^{2}\left\|x_{k}(t)\right\|^{2}\right)+\breve{z}_{2}^{T} u \\
& +\frac{1}{4} \mathcal{z}_{2}^{T} \breve{z}_{2}+\varrho \bar{T}\left(x_{1}\left(t-\vartheta_{1}(t)\right), x_{2}\left(t-\vartheta_{2}(t)\right)\right) \\
& -\sum_{i=1}^{2} \frac{1}{\beta} \widetilde{W}_{i} \Gamma_{i}^{-1} \dot{\widehat{W}}_{i} .
\end{aligned}
$$

Design the actual controller $u$ by the following:

$$
u=Y\left(\breve{z}_{2}\right)\left(-k_{3} \breve{z}_{2}-\frac{\breve{z}_{2}}{2 \eta}-\frac{\breve{z}_{2}}{4 \varrho}-\widehat{W}^{T} S(Z)\right) \text {, }
$$

where $k_{3}=\operatorname{diag}\left\{k_{31}, k_{32}\right\}, k_{3 i}, i=1,2$, represent positive constant, and $\eta=\operatorname{diag}\left\{\eta_{1}, \eta_{2}\right\}$.

Adding (30) into (29) when $\breve{z}_{2} \neq[0,0]^{T}$, it is rewritten as

$$
\begin{aligned}
\dot{V}_{2} \leq & -\sum_{i=1}^{2} k_{1 i} \breve{z}_{1 i}^{2}+\breve{z}_{1}^{T} \breve{z}_{2}+\sum_{i=1}^{2} \breve{z}_{2 i} \widetilde{W}_{i} S_{i}(Z)+\frac{1}{2} \sum_{i=1}^{2} \eta \bar{\varepsilon}_{i}^{2}-\sum_{k=1}^{2} \exp \left(\vartheta_{k}(t)\right)\left(q_{k}^{2}\left\|x_{k}(t)\right\|^{2}\right) \\
& -\sum_{i=1}^{2} k_{3 i} \breve{z}_{2 i}^{2}+\varrho \bar{T}^{2}\left(x_{1}\left(t-\vartheta_{1}(t)\right), x_{2}\left(t-\vartheta_{2}(t)\right)\right)-\sum_{i=1}^{2} \frac{1}{\beta} \widetilde{W}_{i} \Gamma_{i}^{-1} \dot{\hat{W}}_{i} .
\end{aligned}
$$


The adaptation law is considered in the following:

$$
\dot{\widehat{W}}_{i}=Y\left(\breve{z}_{2}\right) \beta \Gamma_{i}\left(\breve{z}_{2 i} S_{i}(Z)-\sigma_{i} \widehat{W}_{i}\right),
$$

where $\sigma_{i}$ represents the positive constant.

Adding (32) into (31) when $\breve{z}_{2} \neq[0,0]^{T}$, (31) will become

$$
\begin{aligned}
\dot{V}_{2} \leq & -\sum_{i=1}^{2} k_{1 i} \breve{z}_{1 i}^{2}+\breve{z}_{1}^{T} \breve{z}_{2}-\sum_{i=1}^{2} k_{3 i} \breve{z}_{2 i}^{2}+\frac{1}{2} \sum_{i=1}^{2} \eta \bar{\varepsilon}_{i}^{2}-\sum_{k=1}^{2} \exp \left(\vartheta_{k}(t)\right)\left(q_{k}^{2}\left\|x_{k}(t)\right\|^{2}\right) \\
& +\sum_{i=1}^{2} \sigma_{i} \widetilde{W}_{i} \widehat{W}_{i}+\varrho \bar{T}^{2}\left(x_{1}\left(t-\vartheta_{1}(t)\right), x_{2}\left(t-\vartheta_{2}(t)\right)\right) .
\end{aligned}
$$

On the basis of the term $\sum_{i=1}^{2} \sigma_{i} \widetilde{W}_{i} \widehat{W}_{i}$ and the equation $\widehat{W}_{i}=W_{i}^{*}-\widetilde{W}_{i}$, we can obtain the following:

$$
\sum_{i=1}^{2} \sigma_{i} \widetilde{W}_{i} \widehat{W}_{i}=\sum_{i=1}^{2} \sigma_{i} \widetilde{W}_{i} W_{i}^{*}-\sum_{i=1}^{2} \sigma_{i}\left\|\widetilde{W}_{i}\right\|^{2} .
$$

According to Young's inequality, we get

$$
\sum_{i=1}^{2} \sigma_{i} \tilde{W}_{i} W_{i}^{*} \leq \frac{1}{2} \sum_{i=1}^{2} \sigma_{i}\left\|\tilde{W}_{i}\right\|^{2}+\frac{1}{2} \sum_{i=1}^{2} \sigma_{i}\left\|W_{i}^{*}\right\|^{2} .
$$

$$
\begin{aligned}
\dot{V}_{2} \leq & -\sum_{i=1}^{2} k_{1 i} \breve{z}_{1 i}^{2}+\breve{z}_{1}^{T} \breve{z}_{2}-\sum_{i=1}^{2} k_{3 i} \breve{z}_{2 i}^{2}-\frac{1}{2} \sum_{i=1}^{2} \sigma_{i}\left\|\widetilde{W}_{i}\right\|^{2}-\sum_{k=1}^{2} \exp \left(\vartheta_{k}(t)\right)\left(q_{k}^{2}\left\|x_{k}(t)\right\|^{2}\right)+\frac{1}{2} \sum_{i=1}^{2} \sigma_{i}\left\|W_{i}^{*}\right\|^{2} \\
& +\frac{1}{2} \sum_{i=1}^{2} \eta \bar{\varepsilon}_{i}^{2}+\varrho \bar{T}^{2}\left(x_{1}\left(t-\vartheta_{1}(t)\right), x_{2}\left(t-\vartheta_{2}(t)\right)\right) .
\end{aligned}
$$

Adding (36) into (33), we can further obtain

On the basis of Assumption 3, we can rewrite the timeSubstituting (38) into (37), we obtain varying delay function $\varrho \bar{T}^{2}(\cdot)$ as

$$
\begin{aligned}
& \varrho \bar{T}^{2}\left(x_{1}\left(t-\vartheta_{1}(t)\right), x_{2}\left(t-\vartheta_{2}(t)\right)\right) \leq \varrho q_{1}^{2}\left\|x_{1}\left(t-\vartheta_{1}(t)\right)\right\|^{2} \\
& \quad+\varrho q_{2}^{2}\left\|x_{2}\left(t-\vartheta_{2}(t)\right)\right\|^{2} .
\end{aligned}
$$

$$
\begin{aligned}
\dot{V}_{2} \leq & -\sum_{i=1}^{2} k_{1 i} \breve{z}_{1 i}^{2}+\breve{z}_{1}^{T} \breve{z}_{2}-\sum_{i=1}^{2} k_{3 i} \breve{z}_{2 i}^{2}-\frac{1}{2} \sum_{i=1}^{2} \sigma_{i}\left\|\widetilde{W}_{i}\right\|^{2}+\varrho q_{1}^{2}\left\|x_{1}\left(t-\vartheta_{1}(t)\right)\right\|^{2}+\varrho q_{2}^{2}\left\|x_{2}\left(t-\vartheta_{2}(t)\right)\right\|^{2} \\
& +\frac{1}{2} \sum_{i=1}^{2} \sigma_{i}\left\|W_{i}^{*}\right\|^{2}+\frac{1}{2} \sum_{i=1}^{2} \eta \bar{\varepsilon}_{i}^{2}-\sum_{k=1}^{2} \exp \left(\vartheta_{k}(t)\right) q_{k}^{2}\left\|x_{k}(t)\right\|^{2} .
\end{aligned}
$$

Choose the LKF candidate as follows: 


$$
V_{l}=Y\left(\breve{z}_{2}\right) \sum_{k=1}^{2}\left(\exp \left(-\left(t-\vartheta_{k}(t)\right)\right) \times \int_{t-\vartheta_{k}(t)}^{t} \exp (s) q_{k}^{2}\left\|x_{k}(s)\right\|^{2} \mathrm{~d} s\right)
$$

Remark 4. For $\breve{z}_{2} \neq[0,0]^{T}$, we think about Remark 3 and the piecewise function $Y\left(\bar{z}_{2}\right)$ at the same time and use LKFs to fully eliminate the positive continuous functions. The LKFs are very effective way to settle the question when there are unknown time-varying delays in the system.

Then, $\dot{V}_{l}$ can be expressed as

$$
\begin{aligned}
\dot{V}_{l}= & \sum_{k=1}^{2}-\left(1-\dot{\vartheta}_{k}(t)\right)\left(\exp \left(-\left(t-\vartheta_{k}(t)\right)\right) \times \int_{t-\vartheta_{k}(t)}^{t} \exp (s) q_{k}^{2}\left\|x_{k}(s)\right\|^{2} d s\right) \\
& -\sum_{k=1}^{2}\left(1-\dot{\vartheta}_{k}(t)\right)\left(q_{k}\left\|x_{k}\left(t-\vartheta_{k}(t)\right)\right\|\right)^{2}+\sum_{k=1}^{2} \exp (\vartheta(t)) q_{k}^{2}\left\|x_{k}(t)\right\|^{2} .
\end{aligned}
$$

On the basis of $\dot{\vartheta}_{k}(t) \leq \vartheta \leq 1$ and $\varrho=1-\vartheta$, we get $-\left(1-\dot{\vartheta}_{k}(t)\right) \leq-(1-\vartheta)=-\varrho$. Furthermore, (41) can be redescribed as

$$
\begin{aligned}
\dot{V}_{l} \leq & -\varrho\left(q_{1}^{2}\left\|x_{1}\left(t-\vartheta_{1}(t)\right)\right\|^{2}+q_{2}^{2}\left\|x_{2}\left(t-\vartheta_{2}(t)\right)\right\|^{2}\right)-\varrho V_{l} \\
& +\sum_{k=1}^{2} \exp \left(\vartheta_{k}(t)\right) q_{k}^{2}\left\|x_{k}(t)\right\|^{2} .
\end{aligned}
$$

Construct the Lyapunov function candidate as follows:

$$
V=V_{2}+V_{l} \text {. }
$$

According to (39), (42), and (43), the derivative of $V$ is redescribed as

$$
\begin{aligned}
\dot{V} \leq & -\sum_{i=1}^{2} k_{1 i} \breve{z}_{1 i}^{2}+\breve{z}_{1}^{T} \breve{z}_{2}-\sum_{i=1}^{2} k_{3 i} \breve{z}_{2 i}^{2}+\frac{1}{2} \sum_{i=1}^{2} \eta \bar{\varepsilon}_{i}^{2}-\varrho V_{l} \\
& -\frac{1}{2} \sum_{i=1}^{2} \sigma_{i}\left\|\widetilde{W}_{i}\right\|^{2}+\frac{1}{2} \sum_{i=1}^{2} \sigma_{i}\left\|W_{i}^{*}\right\|^{2} .
\end{aligned}
$$

On the basis of Young's inequality, we can easily obtain

$$
\breve{z}_{1}^{T} \breve{z}_{2} \leq \frac{1}{2} \breve{z}_{1}^{T} \breve{z}_{1}+\frac{1}{2} \breve{z}_{2}^{T} \breve{z}_{2}
$$

Substituting (45) into (44), we get

$$
\dot{V} \leq-\sum_{i=1}^{2} \breve{k}_{1 i} \widetilde{z}_{1 i}^{2}-\sum_{i=1}^{2} \breve{k}_{2 i} \widetilde{z}_{2 i}^{2}-\varrho V_{l}-\frac{1}{2} \sum_{i=1}^{2} \sigma_{i}\left\|\widetilde{W}_{i}\right\|^{2}+\frac{1}{2} \sum_{i=1}^{2} \sigma_{i}\left\|W_{i}^{*}\right\|^{2}+\frac{1}{2} \sum_{i=1}^{2} \eta \bar{\varepsilon}_{i}^{2},
$$

where $\breve{k}_{1 i}$ and $\breve{k}_{2 i}, i=1,2$, represent positive constant, $k_{1 i}=k_{1 i}-(1 / 2)$, and $k_{2 i}=k_{3 i}-(1 / 2)$.

Then, inequation (46) can be rewritten as

$$
\dot{V} \leq-\kappa V+\phi \text {, }
$$

where

$$
\begin{aligned}
& \kappa=\min \left\{2 \breve{k}_{11}, 2 \breve{k}_{12}, 2 \breve{k}_{21}, 2 \breve{k}_{22}, \varrho, \sigma_{i} \varsigma_{\min }\left(\Gamma_{i}\right), i=1,2\right\}, \\
& \phi=\frac{1}{2} \sum_{i=1}^{2} \sigma_{i}\left\|W_{i}^{*}\right\|^{2}+\frac{1}{2} \sum_{i=1}^{2} \eta \bar{\varepsilon}_{i}^{2} .
\end{aligned}
$$

The parameter selection of the above controller design process directly affects the control performance. And, the control performance can be improved by increasing the design parameter $k_{1}$ and decreasing the design parameters $\eta$, $\sigma_{1}$, and $\sigma_{2}$.

Theorem 1. Assumptions 1-3, the virtual control law (10), the actual controller (30), and the adaptive law (32) are considered for the 2-joint rigid manipulator with unknown time-varying delays; by selecting appropriate parameters, the tracking errors can converge to a compact neighborhood with respect to zero, and all signals of the closed-loop system are semiglobal uniformly ultimately bounded.

Proof. We will discuss two cases when $\breve{z}_{2} \neq[0,0]^{T}$ and $\breve{z}_{2}=[0,0]^{T}$.

Case 1: for $\breve{z}_{2} \neq[0,0]^{T}$, we can obtain 


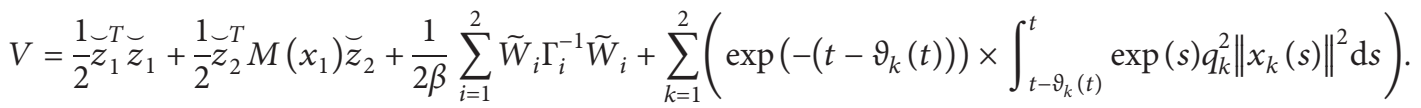

Let us multiply both sides of (47) by $e^{\kappa t}$; we can obtain

$$
\frac{\mathrm{d}\left(V(t) e^{\kappa t}\right)}{\mathrm{d} t} \leq \phi e^{\kappa t}
$$

Then, integrating (50) from $[o, t],(50)$ will become

$$
V(t) \leq\left(V(0)-\frac{\phi}{\kappa}\right) e^{-\kappa t}+\frac{\phi}{\kappa} \leq V(0) e^{-\kappa t}+\frac{\phi}{\kappa} \text {. }
$$

Obviously, each of the terms of (49) is greater than zero, so we can get the following inequality:

$$
\frac{1}{2} \bar{z}_{1}^{T} \breve{z}_{1} \leq V(t) \leq V(0) e^{-\kappa t}+\frac{\phi}{\mathcal{\kappa}} .
$$

On the basis of (49), we easily obtain

$$
\begin{aligned}
V(0)= & \frac{1}{2} \breve{z}_{1}^{T}(0) \breve{z}_{1}(0)+\frac{1}{2} \breve{z}_{2}^{T}(0) \breve{z}_{2}(0)+\frac{1}{2 \beta} \sum_{i=1}^{2} \varsigma_{\max }\left(\Gamma_{i}^{-1}\right)\left\|\widehat{W}_{i}(0)-W_{i}^{*}\right\| \\
& +\sum_{k=1}^{2}\left(\exp \left(-\left(0-\vartheta_{k}(0)\right)\right) \times \int_{0-\vartheta_{k}(0)}^{0} \exp (s)\left(q_{k}^{2} \| x_{k}\left(s \|^{2}\right) d s\right)\right.
\end{aligned}
$$

Next, we can obtain

$$
-D_{1} \leq \breve{z}_{1 i} \leq D_{1}
$$

where $D_{1}=\sqrt{2\left(V(0) e^{-\kappa t}+\phi / \kappa\right)}$.

Then, we can get the compact set of $\breve{z}_{1}$ :

$\Omega_{1}=\left\{\breve{z}_{1} \in R^{2} \mid\left\|\breve{z}_{1 i}\right\| \leq \sqrt{2\left(V(0) e^{-\kappa t}+\frac{\phi}{\kappa}\right)}, i=1,2\right\}$.

At the same time, we can obtain

$$
\frac{1}{2} \bar{z}_{2}^{T} M\left(x_{1}\right) \breve{z}_{2} \leq V(0) e^{-\kappa t}+\frac{\phi}{\kappa} \text {. }
$$

Next, we have

$$
-D_{2} \leq \breve{z}_{2 i} \leq D_{2}
$$

where $D_{2}=\sqrt{V(0) e^{-\kappa t}+(\phi / \kappa) / \varsigma_{\min }\left(M\left(x_{1}\right)\right)}$.

The compact set of $\widetilde{z}_{2}$ can be obtained as

$$
\Omega_{2}=\left\{\breve{z}_{2} \in R^{2} \mid\left\|\breve{z}_{2 i}\right\| \leq \sqrt{\frac{V(0) e^{-\kappa t}+(\phi / \kappa)}{\varsigma_{\min }\left(M\left(x_{1}\right)\right)}}, i=1,2\right\} .
$$

Case 2: for $\breve{z}_{2}=[0,0]^{T}$, we can get controller $u=0$, adaptive law $\widehat{W}_{i}=0$, and LKFs $V_{l}=0$, so (49) will be rewritten as

$$
V=\frac{1}{2} \bar{z}_{1}^{T} \breve{z}_{1}
$$

The derivative of $V$ is expressed in the following:

$$
\dot{V}=\breve{z}_{1}^{T} \dot{z}_{1} \text {. }
$$

On the basis of (11), we can obtain

$$
\dot{V}=-\sum_{i=1}^{2} k_{1 i} \breve{z}_{1 i}^{2} \leq 0 .
$$

According to functional monotony, we can easily get

$$
V(t) \leq V(0) .
$$

Then, (62) can be expressed as

$$
\frac{1}{2} z_{1}^{T} \breve{z}_{1} \leq V(0)
$$

On the basis of (59), we can get

$$
V(0)=\frac{1}{2} \widetilde{z}_{1}^{T}(0) \breve{z}_{1}(0)
$$

Then, we can further have

$$
-D_{3} \leq \breve{z}_{1 i} \leq D_{3},
$$

where $D_{3}=\sqrt{2 V(0)}$.

The compact set of $\breve{z}_{2}$ can be obtained as

$$
\Omega_{3}=\left\{\breve{z}_{1} \in R^{2} \mid\left\|\breve{z}_{1 i}\right\| \leq \sqrt{2 V(0)}, i=1,2\right\} .
$$

\section{Simulation Example}

The dynamic model for the two-joint robotic manipulator with unknown time-varying delay is expressed as

$$
\begin{aligned}
& M(q) \ddot{q}+C(q, \dot{q}) \dot{q}+T\left(q\left(t-\vartheta_{1}(t)\right), \dot{q}\left(t-\vartheta_{2}(t)\right)\right)+G(q) \\
& \quad=u-J^{T}(q) p(t) .
\end{aligned}
$$

Choose the gravitational force as follows: 


$$
\begin{aligned}
G(q) & =\left[G_{11}, G_{21}\right]^{T}, \\
G_{11} & =\left(m_{a} L_{c b}+m_{b} L_{a}\right) g \cos q_{1}+m_{b} L_{c b} g \cos \left(q_{1}+q_{2}\right), \\
G_{21} & =m_{b} L_{c b} g \cos \left(q_{1}+q_{2}\right) .
\end{aligned}
$$

Consider the inertia matrix as

$$
\begin{aligned}
M(q) & =\left[\begin{array}{ll}
M_{11} & M_{12} \\
M_{21} & M_{22}
\end{array}\right]^{T}, \\
M_{11} & =m_{b}\left(L_{a}^{2}+L_{c b}^{2}+2 L_{a} L_{c b} \cos q_{2}\right)+m_{a} L_{c a}^{2}+I_{a}+I_{b}, \\
M_{12} & =m_{b}\left(L_{c b}^{2}+L_{a} L_{c b} \cos q_{2}\right)+I_{b}, \\
M_{21} & =m_{b}\left(L_{c b}^{2}+L_{a} L_{c b} \cos q_{2}\right)+I_{b}, \\
M_{22} & =m_{b} L_{c b}^{2}+I_{b} .
\end{aligned}
$$

The centripetal and Coriolis torques are selected from the following:

$$
\begin{aligned}
C(q, \dot{q}) & =\left[\begin{array}{ll}
C_{11} & C_{12} \\
C_{21} & C_{22}
\end{array}\right]^{T}, \\
C_{11} & =-m_{b} L_{a} L_{c b} \dot{q}_{2} \sin q_{2}, \\
C_{12} & =-m_{b} L_{a} L_{c b}\left(\dot{q}_{1}+\dot{q}_{2}\right) \sin q_{2}, \\
C_{21} & =m_{b} L_{a} L_{c b} \dot{q}_{2} \sin \dot{q}_{2} . \\
C_{22} & =0 .
\end{aligned}
$$

Choose the reversible Jacobian matrix as

$$
\begin{aligned}
J(q) & =\left[\begin{array}{ll}
J_{11} & J_{12} \\
J_{21} & J_{22}
\end{array}\right]^{T}, \\
J_{11} & =-L_{a} \sin q_{1}+L_{b} \sin \left(q_{1}+q_{2}\right), \\
J_{12} & =-L_{b} \sin \left(q_{1}+q_{2}\right), \\
J_{21} & =L_{a} \cos q_{1}+L_{b} \cos \left(q_{1}+q_{2}\right), \\
J_{22} & =L_{b} \cos \left(q_{1}+q_{2}\right) .
\end{aligned}
$$

The time delay function is chosen as

$$
\begin{aligned}
T(q, \dot{q})= & {\left[T_{1}, T_{2}\right]^{T}, } \\
T_{1}= & \sin \left(q_{1}\left(t-\vartheta_{1}(t)\right)\right) q_{2}\left(t-\vartheta_{2}(t)\right) \\
& +e^{\left(q_{1}\left(t-\vartheta_{1}(t)\right)+q_{2}\left(t-\vartheta_{2}(t)\right)\right)}, \\
T_{2}= & \cos \left(q_{1}\left(t-\vartheta_{1}(t)\right)+q_{2}\left(t-\vartheta_{2}(t)\right)\right) \\
& +q_{1}\left(t-\vartheta_{1}(t)\right) q_{2}\left(t-\vartheta_{2}(t)\right) .
\end{aligned}
$$

The virtual control law is considered as

$$
s_{2}^{0}=-k_{1} z_{1}+\dot{y}_{r} .
$$

Consider the controller $u$ as follows:

$$
u=Y\left(\breve{z}_{2}\right)\left(-k_{3} \breve{z}_{2}-\frac{\breve{z}_{2}}{2 \eta}-\frac{\breve{z}_{2}}{4 \varrho}-\widehat{W}^{T} S(Z)\right) .
$$

The NNs adaptation law is chosen as
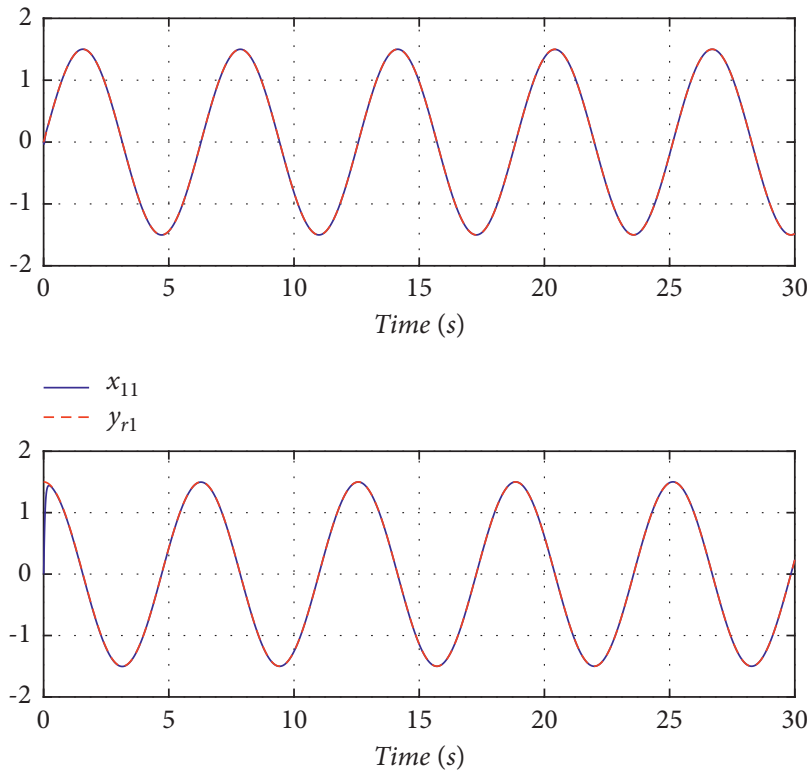

$\begin{array}{ll}- & x_{12} \\ --- & y_{r 2}\end{array}$

FIgURE 1: Trajectories of $x_{1}$ and reference $y_{r}$.

$$
\dot{\hat{W}}_{i}=Y\left(\breve{z}_{2}\right) \beta \Gamma_{i}\left(\breve{z}_{2 i} S_{i}(Z)-\sigma_{i} \widehat{W}_{i}\right) .
$$

The first-order filter is selected as

$$
F(t) \dot{s}_{2}+s_{2}=s_{2}^{0},
$$

where $s_{2}^{0}$ is the input and $s_{2}$ is the output of the first-order filter; $s_{2}(0)=0$ is the initial condition; $F(t)$ is settled as $F(t)=a e^{-\omega t}+c$, and we have the parameters $a>0, \omega>0$, and $c>0$.

In the process of simulation, the initial conditions are $q_{1}(0)=q_{2}(0)=0$ and $\dot{q}_{1}(0)=\dot{q}_{2}(0)=0$; the link mass is $m_{a}=1 \mathrm{~kg}$ and $m_{b}=0.7 \mathrm{~kg}$; the link length is $L_{a}=0.36 \mathrm{~m}$ and $L_{b}=0.32 \mathrm{~m} ; L_{c a}$ and $L_{c b}$ represent the midpoint of link; the link inertia is $I_{a}=56.12 \times 10^{-3} \mathrm{kgm}^{2}$ and $I_{b}=19.78 \times 10^{-3} \mathrm{kgm}^{2}$; the gravitational acceleration is $g=9.81 \mathrm{~m} / \mathrm{s}^{2}$; the external disturbance is selected as $p=[0.5 \sin (t)+1.5,1.5 \cos (t)+0.5]^{T}$; the reference signal stands for $y_{r}=[1.5 \sin (t), 1.5 \cos (t)]^{T}$; the unknown timevarying delays are $\vartheta_{1}(t)=1.5 \sin (0.8 t)$ and $\vartheta_{2}(t)=0.7 \cos (1.2 t)$; the design parameters are selected as $k_{1}=\operatorname{diag}\{19,19\}, \quad k_{3}=\operatorname{diag}\{37,37\}, \quad \eta=\operatorname{diag}\{0.01,0.02\}$, $\varrho=0.02, \sigma_{1}=0.005$, and $\sigma_{2}=0.001$. In the process of simulation, it can be discovered that $k_{1}$ is selected appropriately larger and the design parameter $\eta, \sigma_{1}$, and $\sigma_{2}$ are decreased and there will be good control performance. Neural network nodes are $l_{1}=l_{2}=20, \quad \beta=10$, $\Gamma_{1}=0.5 \operatorname{diag}\{$ ones $\left.(1,20)\}\right]$, and $\Gamma_{2}=0.3 \operatorname{diag}\{$ ones $(1,20)\}$. The first-order filter design parameters are chosen as $a=0.001, \omega=0.001$, and $c=0.001$.

According to the design of the control method, the simulation consequences are shown by Figures 1-3. From Figure 1, we see that the output signal can follow the desired reference signal $y_{r}$. Figure 2 presents the trajectories of $x_{11}$, 


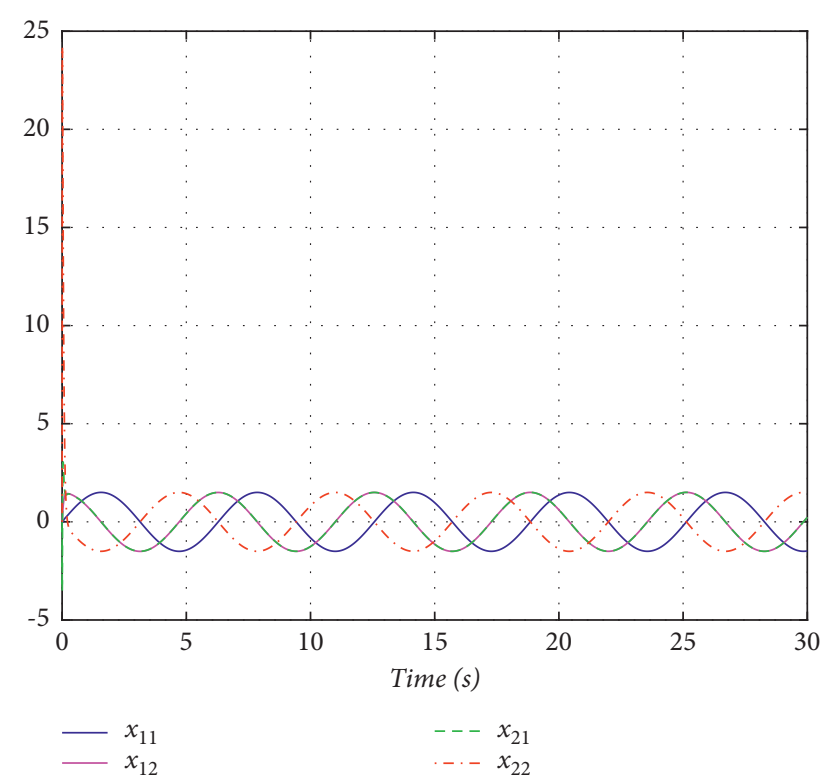

FIGURE 2: Trajectories of $x_{11}, x_{12}, x_{21}$, and $x_{22}$.

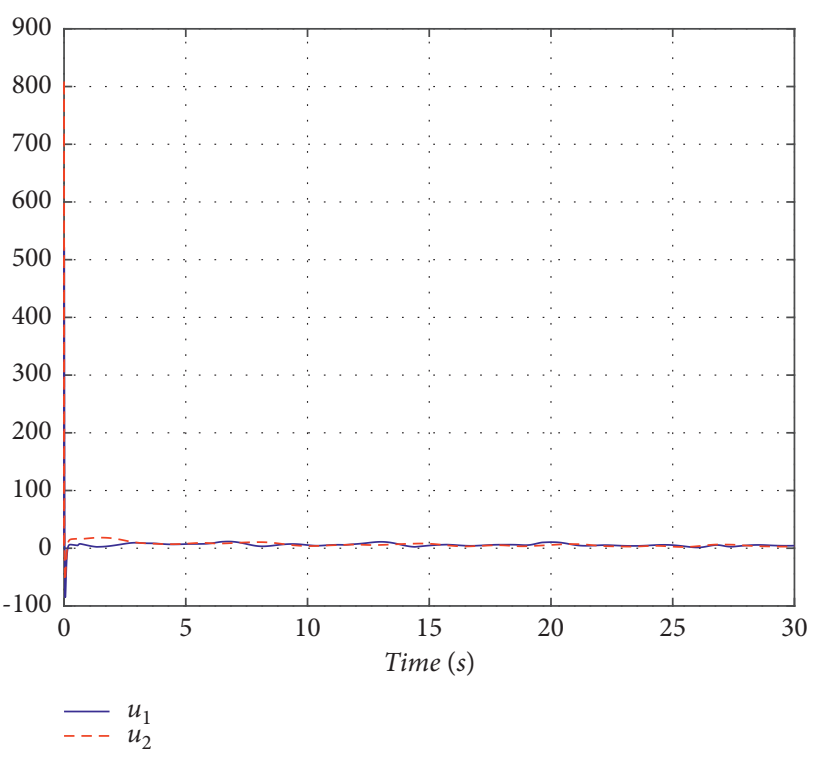

Figure 3: Trajectories of $u$.

$x_{12}, x_{21}$, and $x_{22}$, which illustrate distinctly that all the signals are bounded stable. Figure 3 shows the actual controller. From Figures 1-3, the proposed control method is verified to be effective.

\section{Conclusion}

In this paper, an adaptive neural tracking controller has been investigated for a two-joint rigid manipulator with unknown time-varying delays. The command filter technology is adopted in the traditional backstepping process to avoid repeated derivation of the virtual controller. In order to work out the unknown time-varying delay issue in two-joint rigid manipulators, a method that combines separation technology with LKFs is proposed. The piecewise function is constructed with the aim to settle the singularity issue in controller design process. By utilizing Lyapunov analysis, it has been proved that the adaptive neural tracking method can guarantee that all signals in the closed-loop system are bounded, and the tracking error can converge to a compact neighborhood with respect to zero. Eventually, the simulation example is verified with the effectiveness of the control approach. In the future, we will study the control method of the robotic manipulator with prespecified tracking accuracy.

\section{Data Availability}

The data used to support the findings of this study are available from the corresponding author upon request.

\section{Conflicts of Interest}

The authors declare that they have no conflicts of interest.

\section{Acknowledgments}

This work was supported by the National Natural Science Foundation of China (61903169) and Liaoning Revitalization Talents Program (XLYC2007182).

\section{References}

[1] M. Chen, S. S. Ge, B. V. E. How, and Y. S. Choo, "Robust adaptive position mooring control for marine vessels," IEEE Transactions on Control Systems Technology, vol. 21, no. 2, pp. 395-409, 2013.

[2] J. E. Refsnes, A. J. Sorensen, and K. Y. Pettersen, "Modelbased output feedback control of slender-body underactuated AUVs: theory and experiments," IEEE Transactions on Control Systems Technology, vol. 16, no. 5, pp. 930-946, 2008.

[3] D. Bustan, N. Pariz, and S. K. Hosseini Sani, "Robust faulttolerant tracking control design for spacecraft under control input saturation," ISA Transactions, vol. 53, no. 4, pp. 1073-1080, 2014.

[4] Y. Li, K. Sun, and S. Tong, "Observer-based adaptive fuzzy fault-tolerant optimal control for SISO nonlinear systems," IEEE Transactions on Cybernetics, vol. 49, no. 2, pp. 649-661, 2019. 
[5] S. Tong, Y. Li, and S. Sui, "Adaptive fuzzy tracking control design for SISO uncertain nonstrict feedback nonlinear systems," IEEE Transactions on Fuzzy Systems, vol. 24, no. 6, pp. 1441-1454, 2016.

[6] R. Lu, J. Tao, P. Shi, H. Su, Z.-G. Wu, and Y. Xu, "Dissipativity-based resilient filtering of periodic Markovian jump neural networks with quantized measurements," IEEE Transactions on Neural Networks and Learning Systems, vol. 29, no. 5, pp. 1888-1899, 2018.

[7] H. Wang, B. Chen, and C. Lin, "Direct adaptive neural control for strict-feedback stochastic nonlinear systems," Nonlinear Dynamics, vol. 67, no. 4, pp. 2703-2718, 2012.

[8] Y. Li, S. Qiang, X. Zhuang, and O. Kaynak, "Robust and adaptive backstepping control for nonlinear systems using RBF neural networks," IEEE Transactions on Neural Networks, vol. 15, no. 3, pp. 693-701, 2004.

[9] Y.-J. Liu, S. Li, S. Tong, and C. L. P. Chen, "Adaptive reinforcement learning control based on neural approximation for nonlinear discrete-time systems with unknown nonaffine dead-zone input," IEEE Transactions on Neural Networks and Learning Systems, vol. 30, no. 1, pp. 295-305, 2019.

[10] J. Wu, X. M. Chen, Q. J. Zhao, J. Li, and Z. G. Wu, “Adaptive neural dynamic surface control with prespecified tracking accuracy of uncertain stochastic nonstrict-feedback systems," IEEE Transactions on Cybernetics, pp. 1-14, 2020.

[11] J. Wu, Z.-G. Wu, J. Li, G. Wang, H. Zhao, and W. Chen, "Practical adaptive fuzzy control of nonlinear pure-feedback systems with quantized nonlinearity input," IEEE Transactions on Systems, Man, and Cybernetics: Systems, vol. 49, no. 3, pp. 638-648, 2019.

[12] A. Al-Mahturi, F. Santoso, M. A. Garratt, and S. G. Anavatti, "A robust self-adaptive interval type-2 tS fuzzy logic for controlling multi-input-multi-output nonlinear uncertain dynamical systems," IEEE Transactions on Systems, Man, and Cybernetics: Systems, vol. 52, no. 1, pp. 655-666, 2022.

[13] J. Yu, P. Shi, J. Liu, and C. Lin, "Neuroadaptive finite-time control for nonlinear MIMO systems with input constraint," IEEE Transactions on Cybernetics, pp. 1-8, 2020.

[14] M. Chen, S. S. Ge, and B. Ren, "Adaptive tracking control of uncertain MIMO nonlinear systems with input constraints," Automatica, vol. 47, no. 3, pp. 452-465, 2011.

[15] Y.-J. Liu, L. Tang, S. Tong, and C. L. P. Chen, "Adaptive NN controller design for a class of nonlinear MIMO discrete-time systems," IEEE Transactions on Neural Networks and Learning Systems, vol. 26, no. 5, pp. 1007-1018, 2015.

[16] H. G. Yingchun Wang and Y. C. Wang, "Stability analysis of Markovian jumping stochastic cohen-grossberg neural networks with mixed time delays," IEEE Transactions on Neural Networks, vol. 19, no. 2, pp. 366-370, 2008.

[17] G. Cui, J. Yu, and Q. G. Wang, "Finite-time adaptive fuzzy control for MIMO nonlinear systems with input saturation via improved command-filtered backstepping," IEEE Transactions on Systems, Man, and Cybernetics: Systems, pp. 1-10, 2021.

[18] Y. Ji, H. Zhou, and Q. Zong, “Adaptive neural network command filtered backstepping control of pure-feedback systems in presence of full state constraints," International Journal of Adaptive Control and Signal Processing, vol. 33, no. 5, pp. 829-842, 2019.

[19] B. Miao and T. Li, "A novel neural network-based adaptive control for a class of uncertain nonlinear systems in strictfeedback form," Nonlinear Dynamics, vol. 79, no. 2, pp. 1005-1013, 2015.
[20] J. Yu, P. Shi, and L. Zhao, "Finite-time command filtered backstepping control for a class of nonlinear systems," Automatica, vol. 92, pp. 173-180, 2018.

[21] J. Yu, P. Shi, C. Lin, and H. Yu, “Adaptive neural command filtering control for nonlinear MIMO systems with saturation input and unknown control direction," IEEE Transactions on Cybernetics, vol. 50, no. 6, pp. 2536-2545, 2020.

[22] Y.-X. Li, "Finite time command filtered adaptive fault tolerant control for a class of uncertain nonlinear systems," Automatica, vol. 106, pp. 117-123, 2019.

[23] Y. Cui, H. Zhang, Y. Wang, and H. Jiang, "A fuzzy adaptive tracking control for MIMO switched uncertain nonlinear systems in strict-feedback form," IEEE Transactions on Fuzzy Systems, vol. 27, no. 12, pp. 2443-2452, 2019.

[24] A. Boulkroune, M. M’Saad, and M. Farza, "Adaptive fuzzy controller for multivariable nonlinear state time-varying delay systems subject to input nonlinearities," Fuzzy Sets and Systems, vol. 164, no. 1, pp. 45-65, 2011.

[25] Q. Zhou, L. Wang, C. Wu, and H. Li, “Adaptive fuzzy tracking control for a class of pure-feedback nonlinear systems with time-varying delay and unknown dead zone," Fuzzy Sets and Systems, vol. 329, pp. 36-60, 2017.

[26] D. Li, C. L. P. Chen, Y.-J. Liu, and S. Tong, "Neural network controller design for a class of nonlinear delayed systems with time-varying full-state constraints," IEEE Transactions on Neural Networks and Learning Systems, vol. 30, no. 9, pp. 2625-2636, 2019.

[27] D. Li and D. Li, "Adaptive tracking control for nonlinear timevarying delay systems with full state constraints and unknown control coefficients," Automatica, vol. 93, pp. 444-453, 2018.

[28] B. Chen, X. Liu, K. Liu, and C. Lin, "Novel adaptive neural control design for nonlinear MIMO time-delay systems," Automatica, vol. 45, no. 6, pp. 1554-1560, 2009.

[29] L. V. Truong, S. D. Huang, V. T. Yen, and P. V. Cuong, "Adaptive trajectory neural network tracking control for industrial robot manipulators with deadzone robust compensator," International Journal of Control, Automation and Systems, vol. 18, no. 9, pp. 2423-2434, 2020.

[30] V. T. Yen, W. Y. Nan, and P. Van Cuong, "Robust adaptive sliding mode neural networks control for industrial robot manipulators," International Journal of Control, Automation and Systems, vol. 17, no. 3, pp. 783-792, 2019.

[31] C. Kwan and F. L. Lewis, "Robust backstepping control of nonlinear systems using neural networks," IEEE Transactions on Systems, Man, and Cybernetics-Part A: Systems and Humans, vol. 30, no. 6, pp. 753-766, 2000.

[32] W. He, Y. Chen, and Z. Yin, "Adaptive neural network control of an uncertain robot with full-state constraints," IEEE Transactions on Cybernetics, vol. 46, no. 3, pp. 620-629, 2016.

[33] W. Sun, S.-F. Su, J. Xia, and V.-T. Nguyen, "Adaptive fuzzy tracking control of flexible-joint robots with full-state constraints," IEEE Transactions on Systems, Man, and Cybernetics: Systems, vol. 49, no. 11, pp. 2201-2209, 2019.

[34] W. He, Y. Dong, and C. Sun, "Adaptive neural impedance control of a robotic manipulator with input saturation," IEEE Transactions on Systems, Man, and Cybernetics: Systems, vol. 46, no. 3, pp. 334-344, 2016.

[35] W. W. Sun, Y. Wu, and X. Y. Lv, "Adaptive neural network control for full-state constrained robotic manipulator with actuator saturation and time-varying delays," IEEE Transactions on Neural Networks and Learning Systems, pp. 1-12, 2021.

[36] W. He, Y. Ouyang, and J. Hong, "Vibration control of a flexible robotic manipulator in the presence of input 
deadzone," IEEE Transactions on Industrial Informatics, vol. 13, no. 1, pp. 48-59, 2017.

[37] H. Zhang, Y. Liu, and Y. Wang, "Observer-based finite-time adaptive fuzzy control for nontriangular nonlinear systems with full-state constraints," IEEE Transactions on Cybernetics, vol. 51, no. 3, pp. 1110-1120, 2021.

[38] D.-P. Li and D.-J. Li, "Adaptive neural tracking control for an uncertain state constrained robotic manipulator with unknown time-varying delays," IEEE Transactions on Systems, Man, and Cybernetics: Systems, vol. 48, no. 12, pp. 2219-2228, 2018.

[39] Y.-C. Chang and M.-F. Wu, "Robust tracking control for a class of flexible-joint time-delay robots using only position measurements," International Journal of Systems Science, vol. 47, no. 14, pp. 3336-3349, 2016. 